\title{
Intraoperative Dexmedetomidine for Reduction of Postoperative Delirium in the Elderly: A Scoping Review
}

Gregory B. Harris, BSN, RN, SRNA; Brooke N. Hatchell, BSN, RN, SRNA; Davelin D. Woodard, BSN, RN, SRNA; and

Dr. Dwayne Accardo, DNP, CRNA

College of Nursing - The University of Tennessee Health Science Center - Memphis, TN

\section{Purpose}

The purpose of this scoping review is to examine intraoperative dexmedetomidine use to prevent postoperative delirium (POD) in the elderly (>60 years).

Specific Aims:

- Compare the effectiveness of dexmedetomidine to opioids in reducing the incidence of postoperative delirium.

- Determine if the use of dexmedetomidine will decrease the occurrence of delirium in the elderly in the first 48 hours after surgery.

- Determine if the use of dexmedetomidine will result in better patient outcomes in the first 48 hours of the postoperative period.

\section{Background}

- Opioid-induced POD is a well-recognized entity leading to increased length of hospital stays, increased hospital costs, and increased morbidity in the elderly.

- Dexmedetomidine is a potential alternative for opioids, as it mitigates

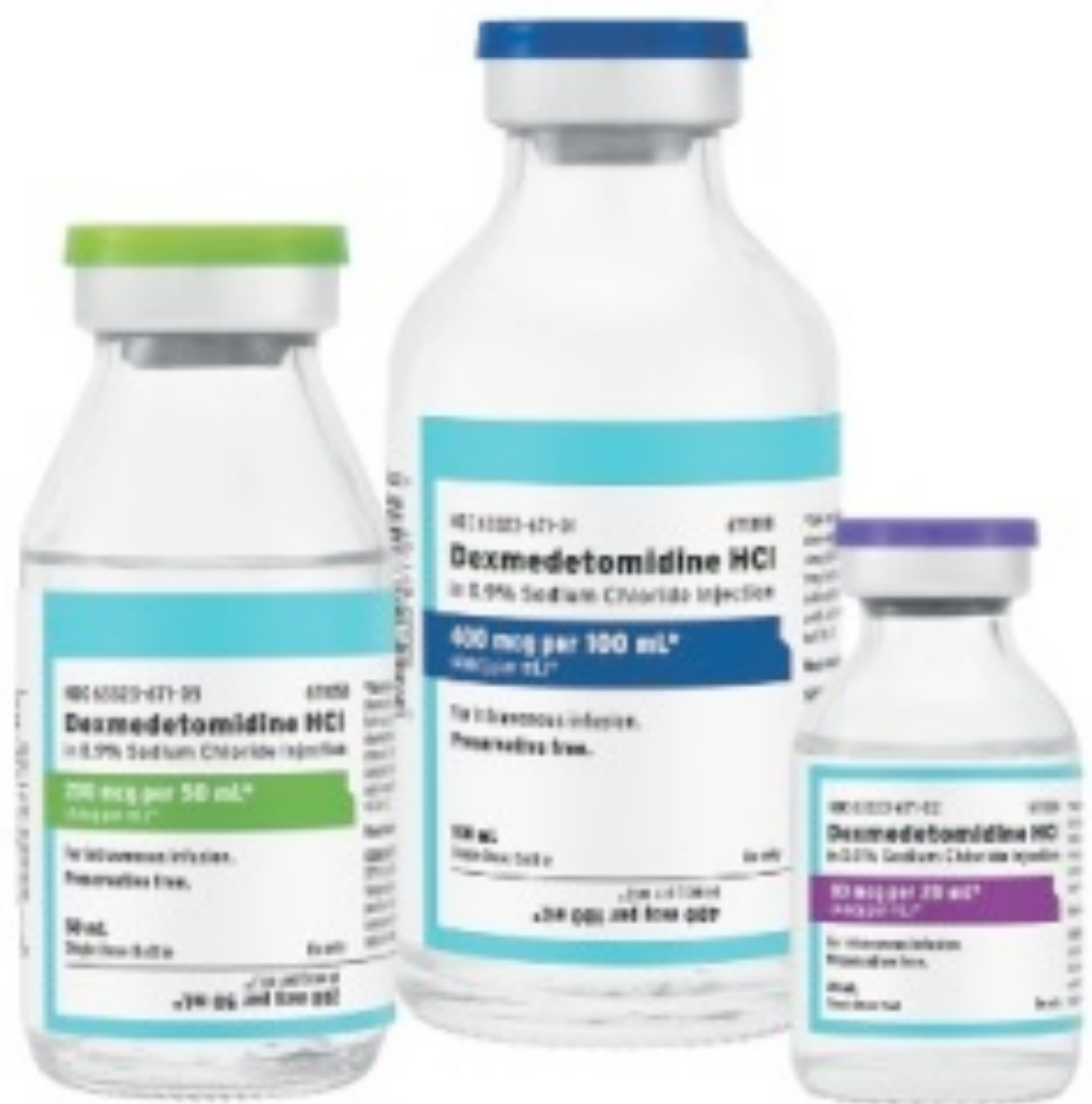
cognitive dysfunction secondary to acute pain compared to standard opioid-based analgesia.

- Dexmedetomidine is an alpha-2 agonist, delivering sedative and analgesic properties with fewer negative effects than opioids.

- Compared to opioids, dexmedetomidine decreases the perception of acute pain, enhances chronic pain management, reduces the frequency of POD, and has no addictive or adverse effects.

\section{Methods}

Eligibility Criteria for Articles

Evaluates the efficacy of dexmedetomidine for prevention

of postoperative delirium

Published in the last 5 years (as of 2019)

Peer-reviewed

Published in English

Abstract included

Free access

Study subjects 60 years or older

The Search

Database search developed with assistance of the academic librarian from November 1 to November 15, 2019.

PubMed, CINAHL, PsychINFO, Google Scholar, and scanning of references

Selection of Sources of Evidence

- 9,000 sources initially identified

Each author selected 5 articles for review by co-authors

Created annotated literature tables for easy screening

4 articles excluded; 11 articles included in scoping review

Data Charting

- Developed data-charting form on Microsoft Word

Data items extracted include author(s) of the publication, the year,

the country of origin, the study population, sample size,

methods/interventions, and key findings relating to the scoping review question

\section{Results}

Synthesis of Results

$81.8 \%$ of the studies advocate for the use of dexmedetomidine in preventing and treating POD and emergence agitation.

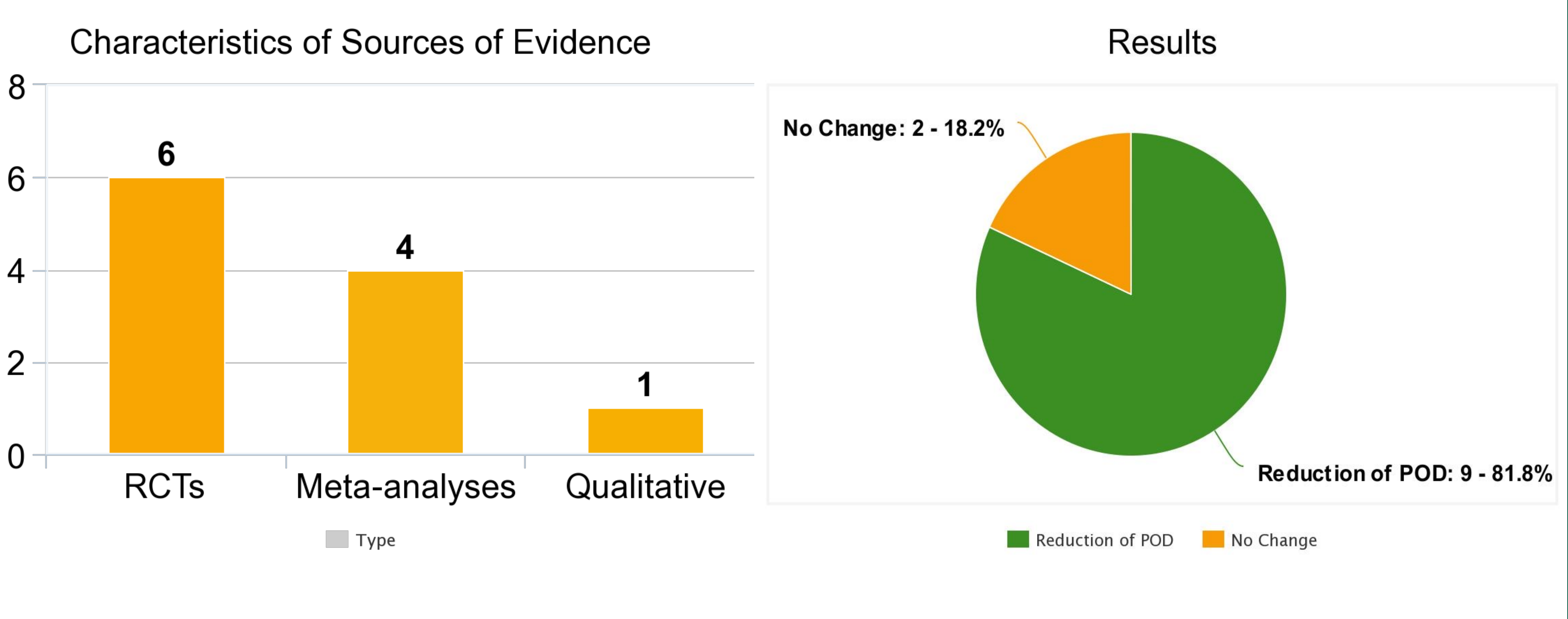

Implications for Practice

- Using the confusion assessment method will aid in identifying those at risk for POD.

- Dexmedetomidine mitigates cognitive impairment induced by acute pain, encouraging early ambulation and shortening length of stay.

- Dexmedetomidine decreases the perception of pain by decreasing the release of norepinephrine. Decreased pain leads to decreased usage of opioids.

- Further research is needed with larger sample sizes, proper screening of patients, and inclusion of providers willing to participate with the study's plan of care.

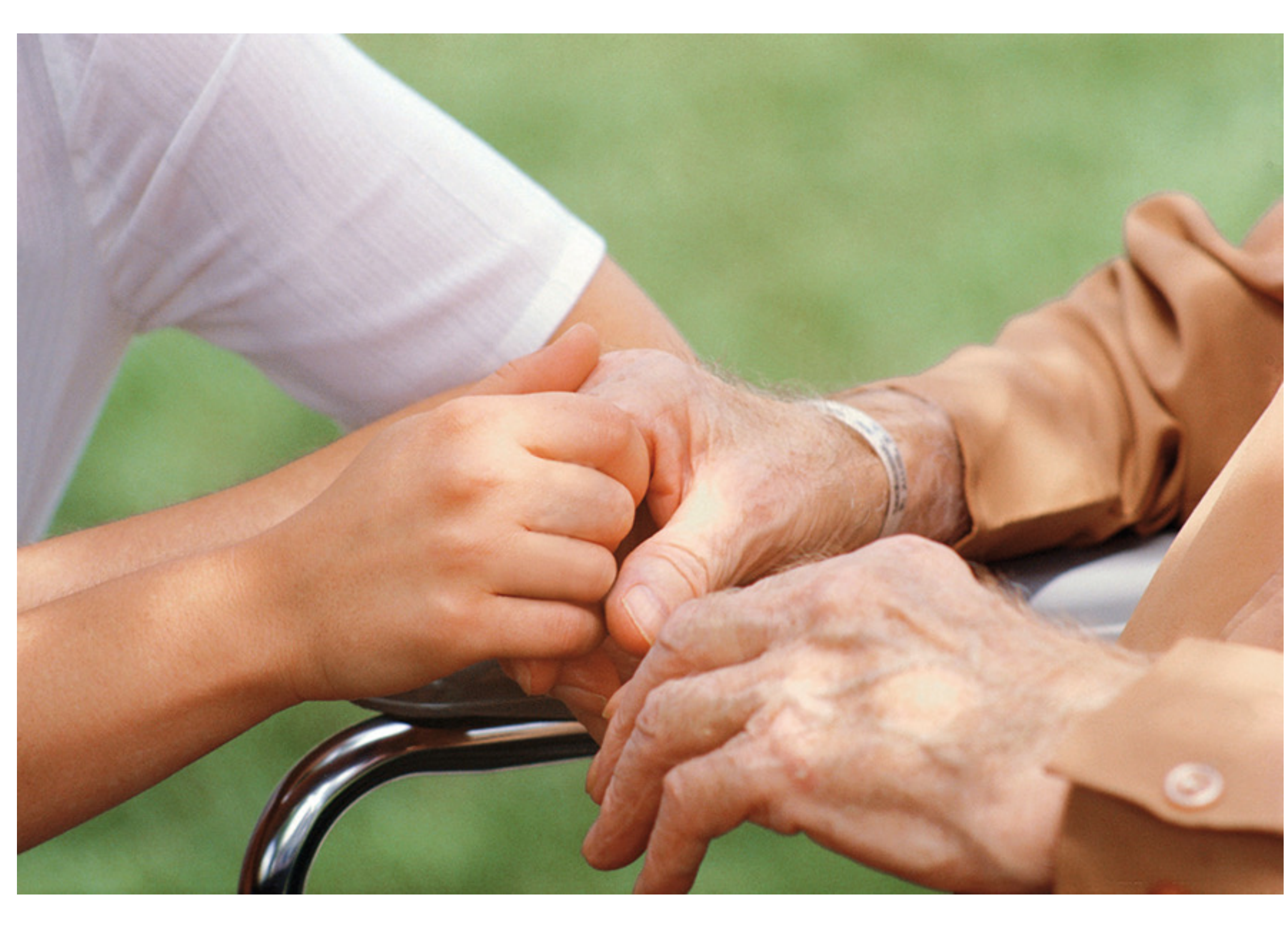

\section{References}

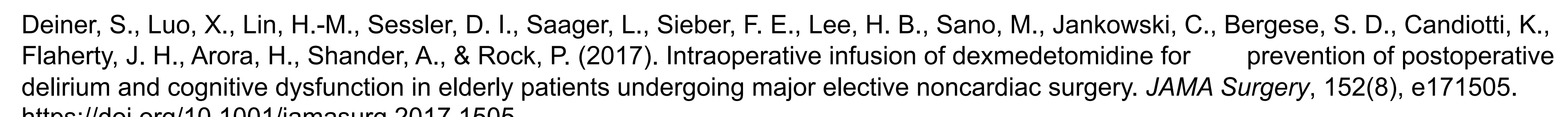

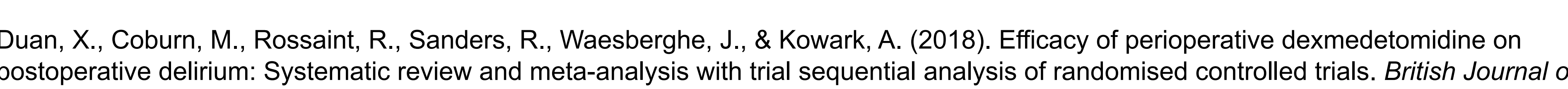

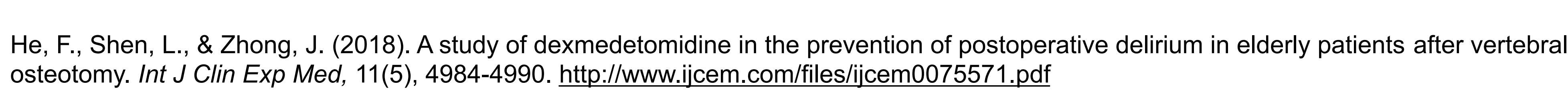

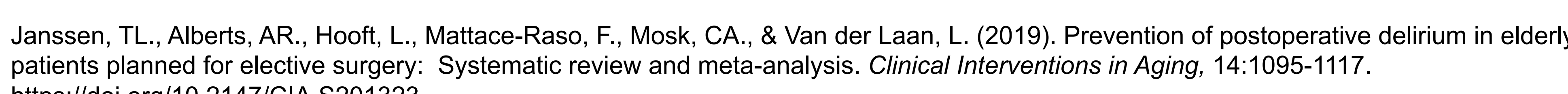

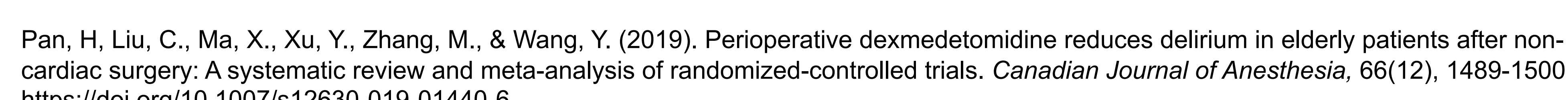

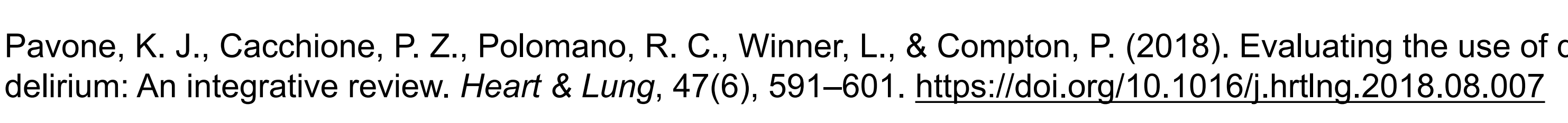

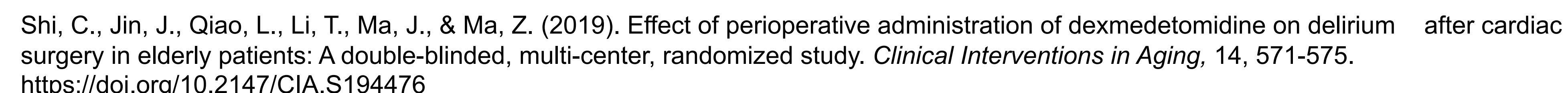

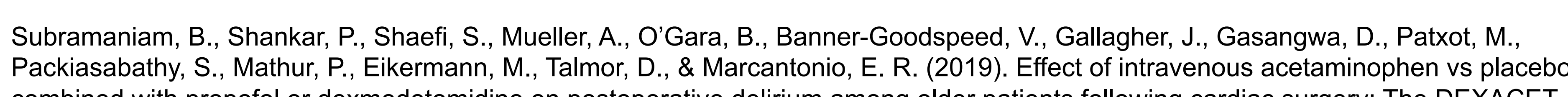

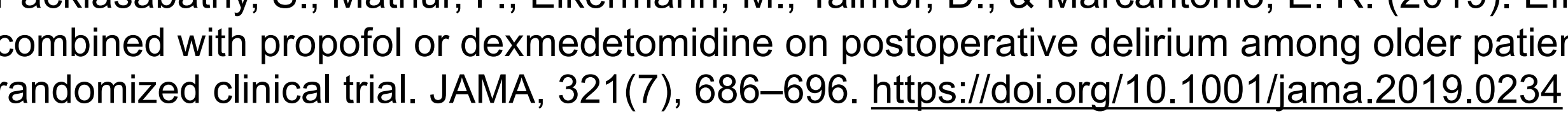

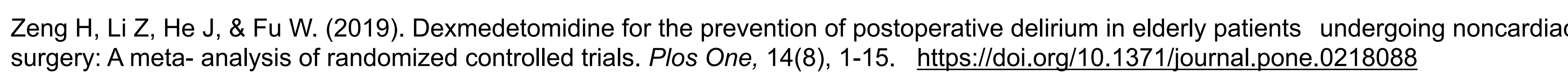

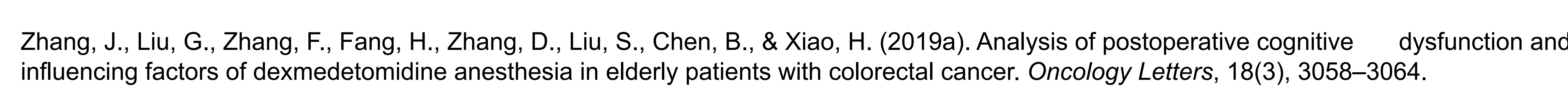

